\title{
The Structure of Cold Molecular Cloud Cores
}

\author{
D. Ward-Thompson \& D. J. Nutter \\ Dept of Physics, Cardiff University, PO Box 913, Cardiff, UK
}

J. M. Kirk

Dept of Astronomy, University of Illinois, Urbana, IL61801, USA

P. André

CEA/DSM/DAPNIA, Service d'Astrophysique, Saclay, France

\begin{abstract}
A brief summary is presented of our current knowledge of the structure of cold molecular cloud cores that do not contain protostars, sometimes known as starless cores. The most centrally condensed starless cores are known as pre-stellar cores. These cores probably represent observationally the initial conditions for protostellar collapse that must be input into all models of star formation. The current debate over the nature of core density profiles is summarised. A cautionary note is sounded over the use of such profiles to ascertain the equilibrium status of cores. The magnetic field structure of pre-stellar cores is also briefly discussed.
\end{abstract}

\section{Introduction}

Low-mass $\left(0.2-2.0 \mathrm{M}_{\odot}\right)$ protostar formation occurs in cold, dense cores within molecular clouds. However, one of the major unknown factors in theories of star formation is a detailed observational determination of the initial conditions of the collapse phase that forms a protostar. There are many different theories of star formation, all predicting different outcomes. Many of the differences can be accredited to the fact that the theories start from different initial collapse conditions. The initial conditions of collapse are crucially important to all models of star formation (for a review, see André, Ward-Thompson \& Barsony 2000).

Many studies of cold cores have been carried out to attempt to determine the initial conditions observationally. Molecular line surveys of dense cores by Myers and co-workers identified a significant number of isolated cores (e.g. Myers \& Benson 1983; Benson \& Myers 1989). Comparison of these surveys with the IRAS point source catalogue detected a class of core that had no associated infrared source. The lack of an embedded source led to the classification of these as 'starless' cores (Beichman et al. 1986).

Ward-Thompson et al. (1994) coined the term pre-protosellar cores to refer to those cores without embedded stars that appear to be sufficiently centrally condensed to be about to form stars. This term has subsequently been shortened 


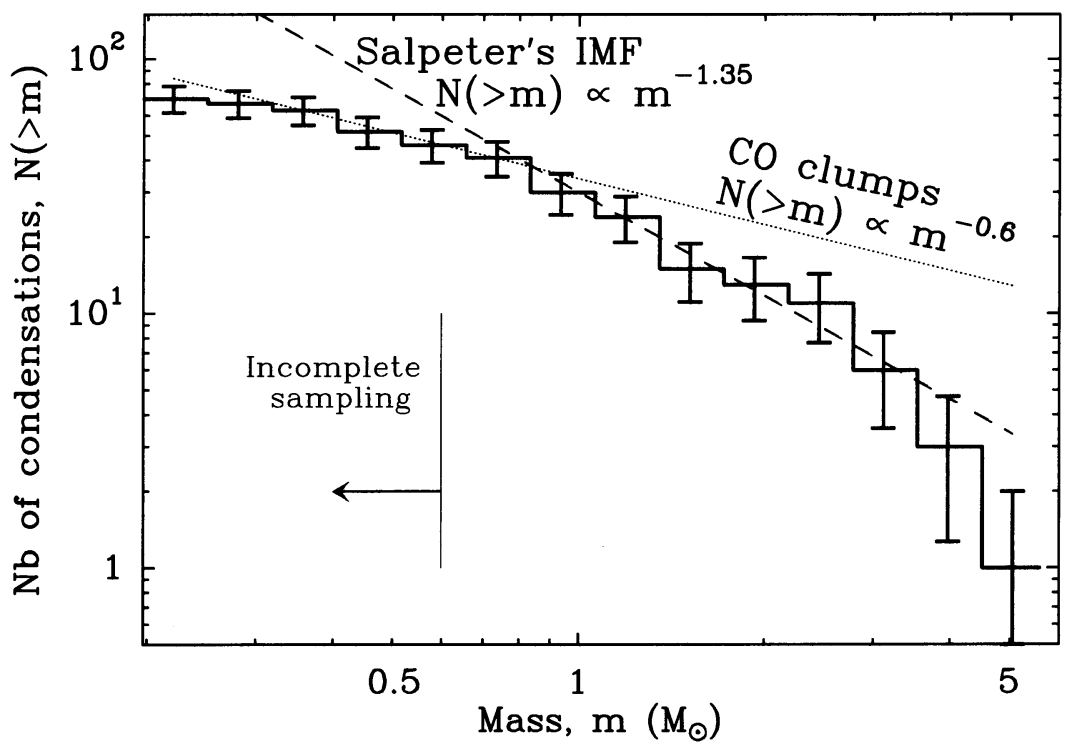

Figure 1. The clump mass distribution of the Orion B molecular cloud (from Motte et al. 2001). Note that the distribution can be fitted by two power-laws, in a manner reminiscent of the stellar IMF. This appears to indicate that the masses of stars are determined strongly by the masses of the pre-stellar cores from which they form.

to 'pre-stellar cores' for brevity. Pre-stellar cores are clearly a key stage on the road to star formation.

Some recent observations have even indicated that the Initial Mass Function (IMF) of stars may be determined at the pre-stellar core stage (Motte, André $\&$ Neri 1998; Motte et al. 2001). Figure 1 shows a plot of the mass distribution of the pre-stellar cores in the Orion B molecular cloud region (from Motte et al. 2001). The form of the distribution mimics the stellar IMF, apparently indicating that the masses of stars are actually determined very early in the star formation process, at the pre-stellar core stage. If this proves to be correct, then in order to understand the cause of the IMF, we must first understand the physics of pre-stellar cores.

\section{Core morphologies}

Figure 2 shows a series of images of the L1544 pre-stellar core at different far-IR and submm wavelengths. The core is barely visible at $90 \mu \mathrm{m}$, but can be seen clearly at longer IR wavelengths. In the submm, the core appears somewhat fainter once again. This all indicates that the core is very cold (see below).

The morphology of L1544 can be seen from Figure 2 to be non-circular. In the higher resolution $850 \mu \mathrm{m}$ data it can even be seen to appear to break up into sub-structure. Nevertheless, many star formation collapse models still start 

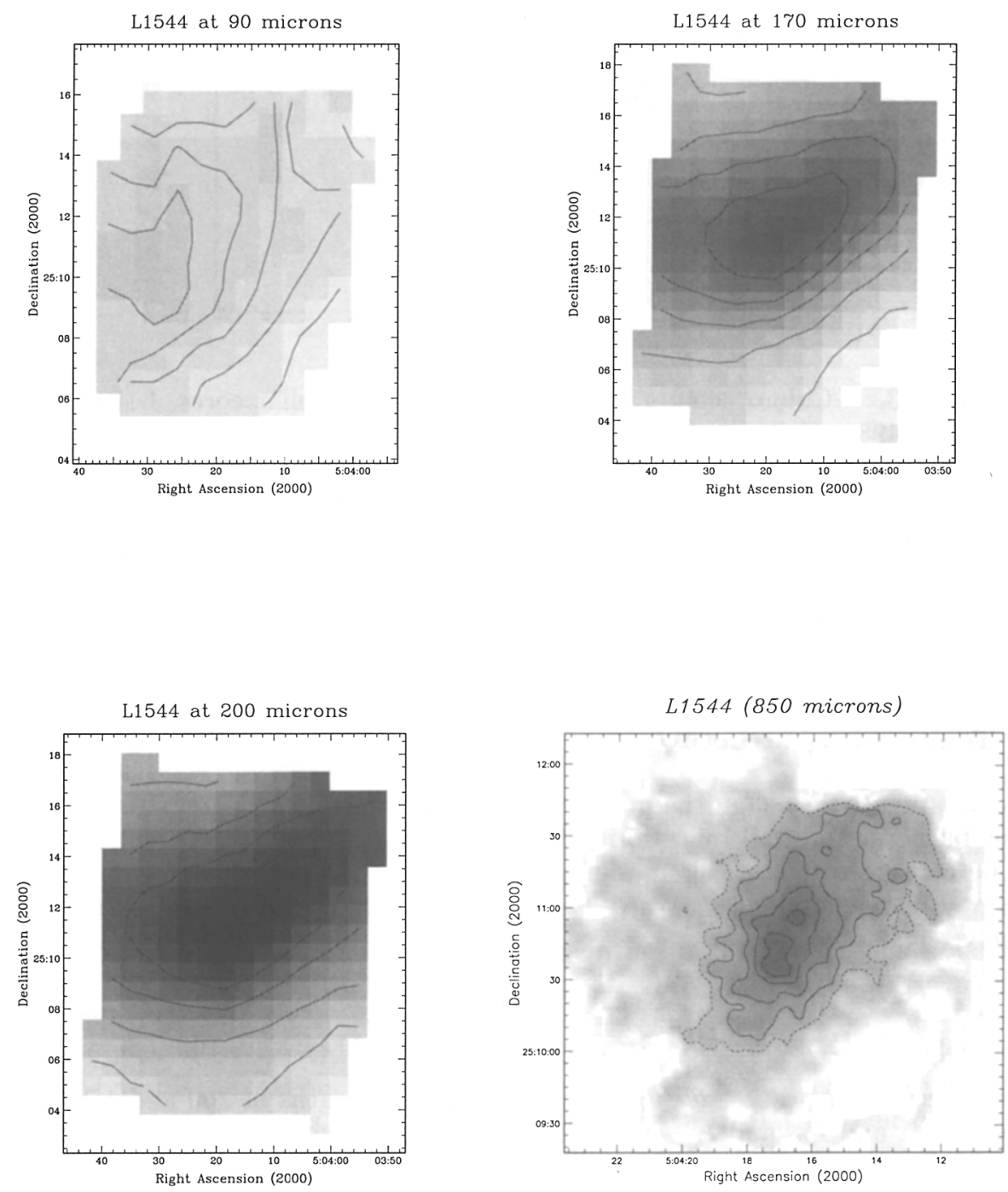

Figure 2. Images of the L1544 molecular cloud core at 90, $170 \&$ $200 \mu \mathrm{m}$ as seen by ISO, and at $850 \mu \mathrm{m}$ as seen by SCUBA on the JCMT. The angular resolution of the $850 \mu \mathrm{m}$ data is higher than that at the other wavelengths. Note that the core is seen most clearly at the longer IR wavelengths, and that it is clearly non-circular. 

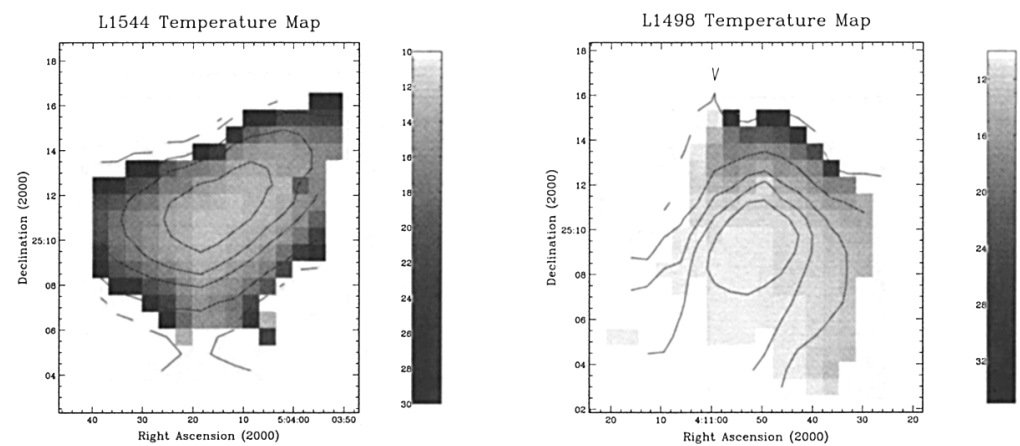

Figure 3. Colour temperature maps of two pre-stellar cores, L1544 and L1498, constructed using the ratio of the 200 and $170 \mu \mathrm{m}$ data. Note that both cores appear warmer at the edge than at the centre. This is consistent with cores that are externally heated, and have not yet formed protostars in their centres.

from spherically symmetric initial conditions. However, some recent studies at least use elliptical averaging to characterise the cores (see below).

\section{Temperature structure}

We can estimate the colour temperature variation across a core (after subtraction of the background level) by ratio-ing the images at 170 and $200 \mu \mathrm{m}$. This ratio can be converted into a colour temperature using the assumption of optically thin grey-body emission (see Ward-Thompson et al. 2002 for further discussion), namely:

$$
\left(F_{\nu_{1}} / F_{\nu_{2}}\right)=\frac{\nu_{1}^{3+\beta}\left(e^{\left[h \nu_{2} / k T\right]}-1\right)}{\nu_{2}^{3+\beta}\left(e^{\left[h \nu_{1} / k T\right]}-1\right)}
$$

where the frequencies $\nu_{1}$ and $\nu_{2}$ correspond to wavelengths of 200 and $170 \mu \mathrm{m}$ respectively, and $F_{\nu_{1}}$ and $F_{\nu_{2}}$ are the flux densities at each of these frequencies. $\mathrm{T}$ is the dust temperature, $\beta$ is the dust emissivity index, and $h$ and $k$ are the Planck and Boltzmann constants respectively. This is one of the simplest sets of assumptions that can be made, as it assumes that all of the dust in a given pixel is at a single temperature, $\mathrm{T}$, and that it is exactly the same dust which is emitting at both wavelengths. We used $\beta=2$ to fit the data, as we found this to be the typical value for these cores (Ward-Thompson et al. 2002). Using these assumptions we constructed a series of colour temperature maps.

Figure 3 shows the colour temperature maps we constructed for two cores, L1544 and L1498. In both cases we see a temperature structure that features a gradient which is cooler at the centre than the edge. This tends to indicate that these cores show the behaviour one would qualitatively expect for externally heated cores, and they do not have a central protostar yet, as was suspected. Hence they are confirmed as pre-stellar in nature. 

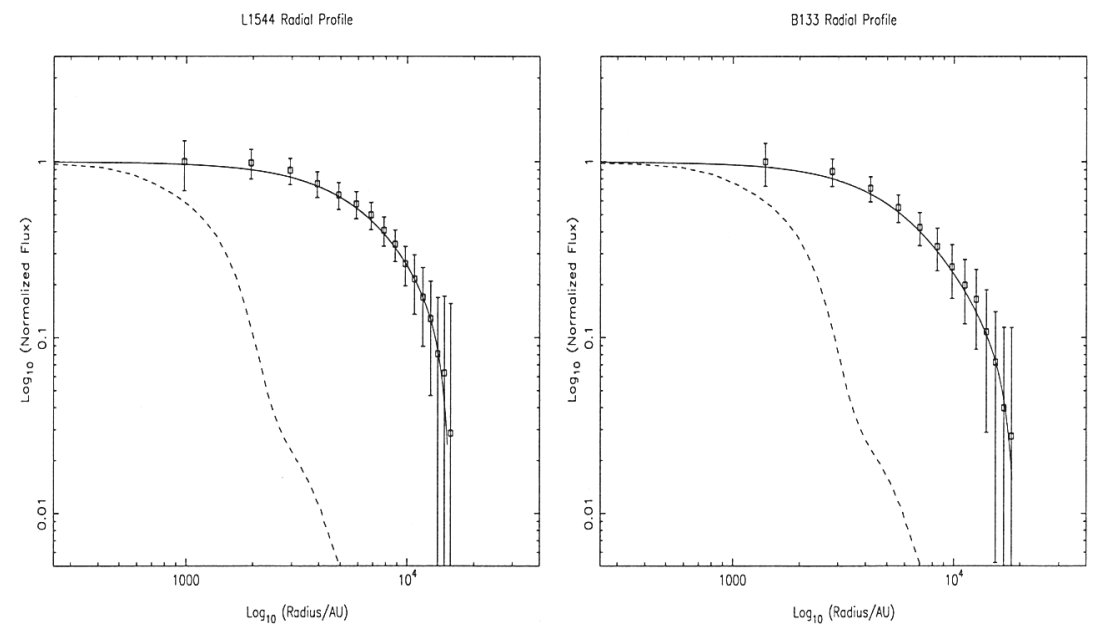

Figure 4. Normalised logarithmic radial flux density profiles of prestellar cores L1544 and B133 (elliptically averaged). The profile is flat in the centre and steepens towards the edge in each case. This is typical of pre-stellar cores and has been modelled in several ways.

\section{Density structure}

Ward-Thompson et al. (1994) were the first to find that pre-stellar cores all appear to follow a form of density profile that is relatively flat in the centre and steeper towards the edge. This is now seen to be a characteristic property of all pre-stellar cores. This profile cannot be explained as simply the result of the temperature profile discussed above, as has been claimed (the observed submm flux density profile is a multiple of temperature and density), since the same shape of profile is seen in mid-IR and near-IR absorption surveys (Bacmann et al. 2000; Alves et al. 2001).

Figure 4 shows the radial flux density profiles of two pre-stellar cores, L1544 and B133, azimuthally averaged in elliptical bins to account for the noncircularity of the cores. The profiles can be seen to be flat in their centres and steeper towards their edges (for further discussion see: Kirk 2002; Kirk et al. 2003). There have been several attempts to characterise this form of profile, in terms of two power-laws (Ward-Thompson et al. 1994), multiple power-laws (Henriksen et al. 1997), Plummer-type profiles (Whitworth \& Ward-Thompson 2001), or Bonnor-Ebert profiles (Alves et al. 2001).

The latter interpretation of density structure in terms of Bonnor-Ebert profiles is interesting, because it might imply that the cores are in some form of pressure equilibrium. However, this result may be misleading. Some recent work has shown that even highly non-equilibrium cores can demonstrate a BonnorEbert form of profile (e.g. Ballesteros-Paredes et al. 2003).

Furthermore, it can be difficult to differentiate between forms of profile based on a two-dimensional representation such as an astronomical image. Recent work by Harvey et al. (2003a) has shown that for some data-sets the profiles 
L183 Polarised Intensity

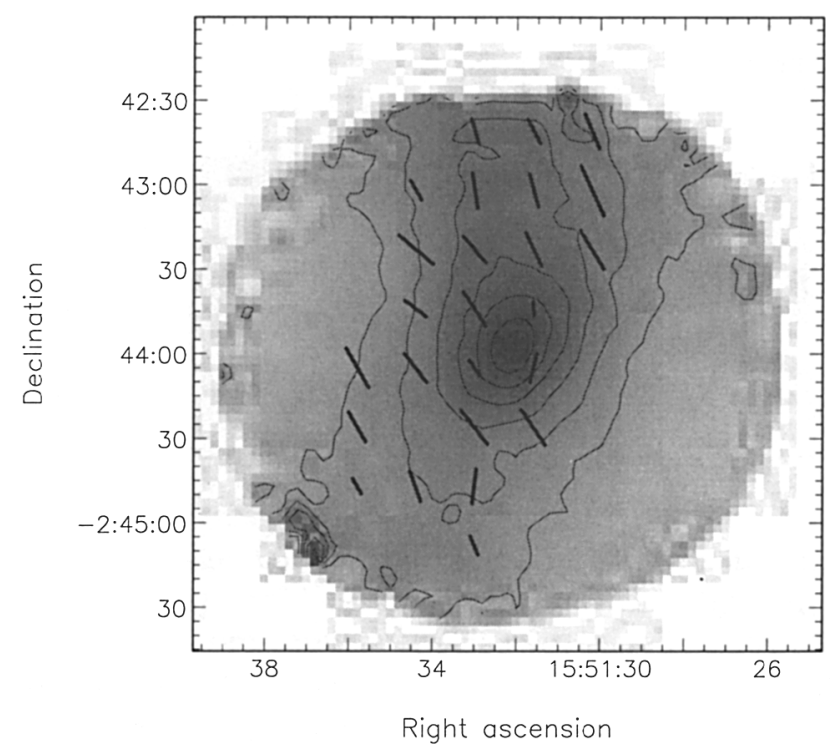

Figure 5. $\quad 850-\mu \mathrm{m}$ image of L183 with polarised intensity vectors superposed taken with SCUBAPOL (from Crutcher et al., 2003). The vectors have been rotated by $90^{\circ}$ to indicate the magnetic field direction. Note that the field is not aligned with either the long axis or the short axis of the core.

can often be fitted by many different forms of profile. This would tend to suggest that interpreting a particular profile in terms of a pressure-balanced equilibrium may be over-interpreting a limited amount of data.

Nonetheless, even if there may be ambiguity over exactly how to model the density profiles, some forms of profile can be ruled out. For instance both the singular isothermal sphere and the logotropic non-isothermal sphere models can be ruled out (Bacmann et al. 2000). The absolute value of the density at the centre and at the edge can also provide information to help differentiate between models.

For example, in some cases a purely thermal Bonnor-Ebert equilibrium model can be ruled because the central temperature predicted by the model is much higher than the observed temperature (e.g. André et al. 2003; Harvey et al. 2003b). This could either be indicating that the cores are already collapsing or that there is some additional form of support that is operating, such as the support of a magnetic field.

\section{Magnetic fields}

The orientation of the magnetic field (in the plane of the sky) in pre-stellar cores can be ascertained from submillimetre polarisation measurements of the cores. Figure 5 shows a polarised intensity map of the L183 pre-stellar core taken using SCUBA with its polarimeter SCUBAPOL (from Crutcher et al. 2003). 
The vectors in Figure 5 have been rotated by $90^{\circ}$ to indicate the B-field direction that can be inferred from the measured polarisation.

It can be seen from Figure 5 that the B-field is not aligned with the short axis of the core, as would be predicted from quasi-static models in which collapse occurs preferentially along the field lines. Nonetheless the field appears moderately uniform across the core, apparently ruling out highly turbulent motions tangling the field lines.

A detailed study of the dispersion of the vector orientations (Crutcher et al. 2003) in this core concluded that the observations are consistent with magnetically-supported models of star formation in a weakly turbulent medium. A comparison with turbulent magneto-hydrodynamic simulations (e.g. Ostriker et al. 1999) leads to similar conclusions (for further discussion see WardThompson et al. 2000). Hence to model the evolution of these cores it appears we need to consider both the magnetic field and turbulence.

\section{Conclusions}

We have given a brief summary of the current state of knowledge of the structure of cold molecular cloud cores, in which solar-mass stars are believed to form. We have concentrated on the properties of pre-stellar cores - those believed to be closest to protostellar collapse. The main points can be summarised as follows:

- Pre-stellar core morphologies are generally not spherically symmetric.

- Typical core temperatures are around $10 \mathrm{~K}$.

- Cores show a tendency to be cooler in their centres than at their edges.

- Core density profiles are flatter at their centres than at their edges.

- Core density profiles can typically be fitted by several different analytic profiles.

- Core density profiles alone cannot be used to determine the equilibrium status of pre-stellar cores.

- Core magnetic fields seem to be relatively uniform, but aligned with neither the core major nor minor axes.

The latter point could be indicating that both turbulence and magnetic fields play a role in the evolution of pre-stellar cores. We support the continued study of pre-stellar cores, as they represent our best opportunity for observing the initial conditions for protostellar collapse.

\section{References}

André, P., Bouwman, J., Belloche, A., \& Hennebelle, P. 2003, in press, in: 'Chemistry as a diagnostic of star formation', eds: Curry, C. L., Fich, M., astro-ph/0212492 
André, P., Ward-Thompson, D., \& Barsony, M. 2000, in: 'Protostars and Planets IV', p. 59, eds: Mannings, V., Boss, A. P., Russell, S. S., University of Arizona Press, Tucson

Alves, J. F., Lada, C. J., \& Lada, E. A. 2001, Nature, 409, 159

Bacmann, A., André, P., Puget, J-L., Abergel, A., Bontemps, S., Ward-Thompson D. $2000, A \& A, 361,555$

Ballesteros-Paredes, J., Klessen, R. S., Vazquez-Semadeni, E. 2003, ApJ, 592, 188

Beichman, C. A., Myers, P. C., Emerson, J. P., Harris, S., Mathieu, R., Benson, P. J., \& Jennings, R. E. 1986, ApJ, 307, 337

Benson, P. J., \& Myers, P. C. 1989, ApJS, 71, 89

Crutcher, R. M., Nutter, D. J., Ward-Thompson, D., \& Kirk, J. M. 2003, ApJ, in press, astro-ph/0305604

Harvey, D. W. A., Wilner, D. J., Myers, P. C., \& Tafalla, M. 2003a, ApJ, in press, astro-ph/0307106

Harvey, D. W. A., Wilner, D. J., Lada, C. J., Myers, P. C., \& Alves, J. F. 2003b, ApJ, in press, astro-ph/0308143

Henriksen, R., André, P., \& Bontemps, S. 1997, A\&A, 323, 549

Kirk, J. M. 2002, PhD Thesis, Cardiff University

Kirk, J. M., Ward-Thompson, D., \& André, P. 2003, in prep

Motte, F., André, P., \& Neri, R. 1998, A\&A, 336, 150

Motte, F., André, P., Ward-Thompson, D., \& Bontemps, S. 2001, A\&A, 372, L41

Myers, P. C., \& Benson, P. J. 1983, ApJ, 266, 309

Ostriker, C. E., Gammie, C. F., \& Stone, J. M. 1999, ApJ, 513, 259

Ward-Thompson, D., André, P., \& Kirk, J. M. 2002, MNRAS, 329, 257

Ward-Thompson, D., Kirk, J. M., Crutcher, R. M., Greaves, J. S., Holland, W. S., \& André, P. 2000, ApJ, 537, L135

Ward-Thompson, D., Scott, P. F., Hills, R. E., \& André, P. 1994, MNRAS, 268, 276

Whitworth, A. P., \& Ward-Thompson, D. 2001, ApJ, 547, 317 